\begin{tabular}{ccc}
\hline & International Journal of Engineering \& Technology, $7(2.12)(2018) 101-104$ \\
SPC & International Journal of Engineering \& Technology \\
Website: $w w w . s c i e n c e p u b c o . c o m / i n d e x . p h p / I J E T$ & Research Paper \\
\hline
\end{tabular}

\title{
DTV broadcasting failover switching system using PCR information
}

\author{
Chang-Hyung Kim ${ }^{1}$, Seong Jeong ${ }^{1}$, Kyoung-Hak Lee ${ }^{2}$, Chae-Bong Sohn ${ }^{\text {** }}$ \\ ${ }^{1}$ Dept. of Electronics and Communications Engineering, Kwangwoon University, 20 Kwangwoon-Ro, \\ Nowon-Gu, Seoul, 01897, South Korea \\ ${ }^{2}$ IACF, Kwangwoon University, 20 Kwangwoon-Ro, Nowon-Gu, Seoul, 01897, South Korea \\ *Corresponding author E-mail: Email: cbsohn@kw.ac.kr
}

\begin{abstract}
Background/Objectives: Major Terrestrial broadcasters, MSO (Multi-System Operator), IPTV (Internet Protocol Television) Operators have been putting effort to prevent the transmission stops such as transmission interruption.

Methods/Statistical analysis: The main and spare signals of the digital broadcasting are monitored. If a problem occurs in the main signal, it automatically switched to a preliminary signal, preventing a broadcast accident before a failover switch is performed.

In this paper, we propose a method that can automatically switch signal to minimize the resulting impacts on a broadcasting accident caused by a disconnection from the input or an accident from the network. Digital broadcasts transmission abides by MPEG-2 (Moving Picture Expert Group) TS (Transport Stream) standardization.

Findings: This paper proposes a method to maintain the quality of broadcast such as transmission interruption within ETSI TR 101290 (European Telecommunications Standards Institute Technical Report) standardization. Furthermore, with this proposed method, it would be possible to minimize the error from an automatically transferred stream that sent from the emergency link within ETSI TR 101290 standardization.
\end{abstract}

Improvements/Applications: A method for preventing broadcast transmission accidents

Keywords: Dtv; Mpeg2; Transport Stream; Pcr; Failover Switch

\section{Introduction}

Recently, terrestrial digital HD broadcasting is provided using ATSC (Advanced Television Systems Committee, Inc.) 8-VSB (Vestigial Side-Band) system through a $6 \mathrm{MHz}$ bandwidth in Korea, and broadcasting signals are retransmitted through facilities such as apartments, buildings, and public house ${ }^{1}$. Therefore, major terrestrial broadcasters, multi-system and IPTV operators have been put effort to prevent the transmission stops such as a transmission interruption. Both main and spare signals of the digital broadcasting are monitored as usual, and when the main signal gets a problem, it automatically switched to a preliminary signal, preventing a broadcast accident before a failover switch is performed. Generally, conventional equipment switches are RF (Radio Frequency), ASI (Asynchronous Serial Interface), and IP (Internet Protocol) inputs. In these cases, the RF signal strength, BER(Bit Error Rate), or MPEG-2 TS header synchronization is examined to forcibly switch when it losses signal a packet error occurs. Then, the emergency switching link could meet a problem due to such physical switching and MPEG-2 TS packet loss. Moreover, the broadcast quality is decreased by an asynchronization between main and spare inputs and a break of an audio and video is deteriorated [2].

In this paper, we propose a method to minimize the influence of broadcast incidents when automatic switching to an emergency link by using PCR (Program Clock Reference) of MPEG-2 TS packet header. This maintains broadcast quality by naturally linking broadcast output conversions. Also, it minimizes errors in ETSI TR 101
290 specification when a broadcast accident or forced switchover occurs [3].

\section{Materials and methods}

\subsection{Dtv transmission overview}

In Korea, digital broadcasting service is conducted based on ATSC 8-VSB modulation method from North American Broadcasting Standards Association[4,5]. The concept of ATSC broadcast transmission is shown in Figure 1.

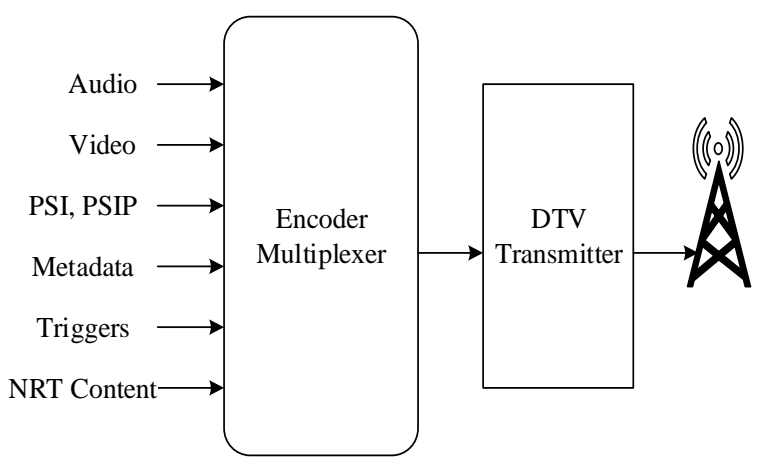

Fig. 1:.ATSC Broadcasting Transmission Concept Diagram. 


\subsection{Mpeg-2 ts}

MPEG-2 TS is a system protocol for audio, video, and data transmission used for digital broadcast transmission.TS supports packet header and the program specific information (PSI).At the receiver, this information is used to detect TS packet errors and analyzes the configuration of the program. The TS packet header and the PSI configuration information are shown in Figure 2 and Figure 3.

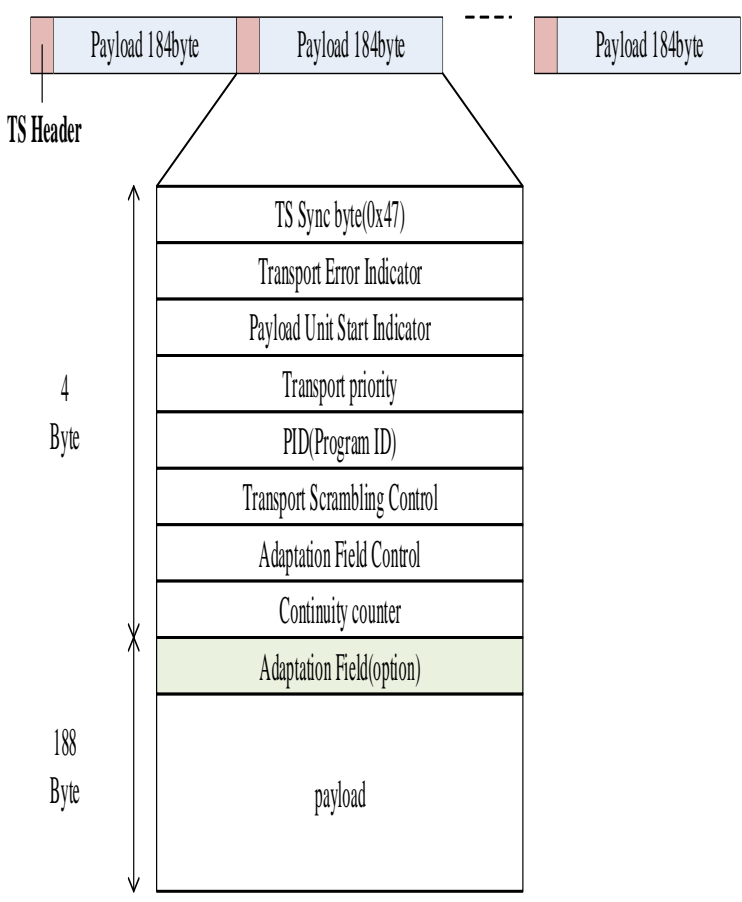

Fig. 2: MPEG-2 TS Packet Structure.

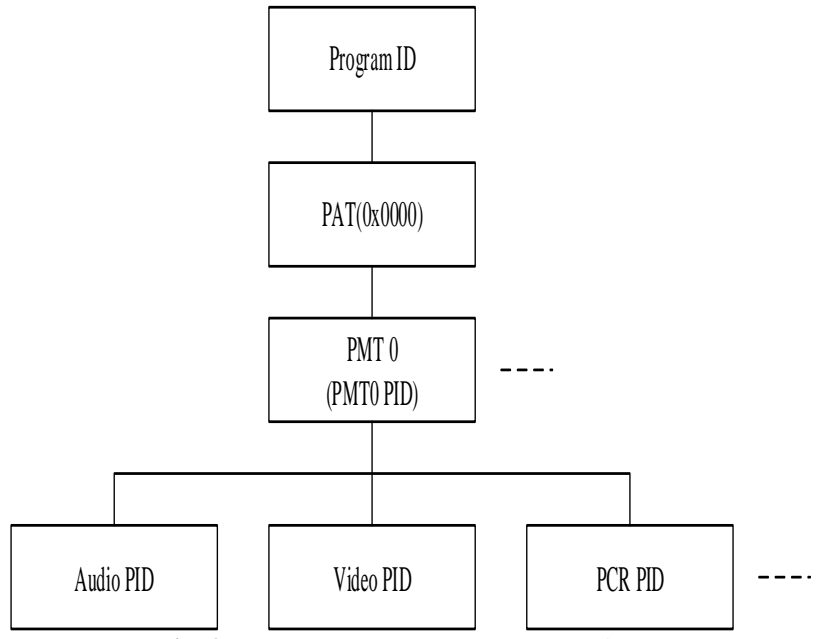

Fig. 3:.MPEG-2 TS Table \&Structure of PSI.

PSI is information representing the configuration of a program included in the MPEG-2 TS. It distinguishes among several programs divides the video, audio, and data of the program; provides information to use the broadcasting service. The PSI components have PAT (Program Association Table), PMT (Program Map Table), CAT (Conditional Access Table), and NIT (Network Information Table). After analyzing PMT through PAT, audio, video, and PCR PID (Program Identity) can be found through PMT.

\subsubsection{Scr \& per}

In the MPEG-2 TS, the transmitter transmits a time reference value from the program to the receiver. With Figure 4 and equations from 1 to 3, the PCR location and the PCR value can be evaluated [6].

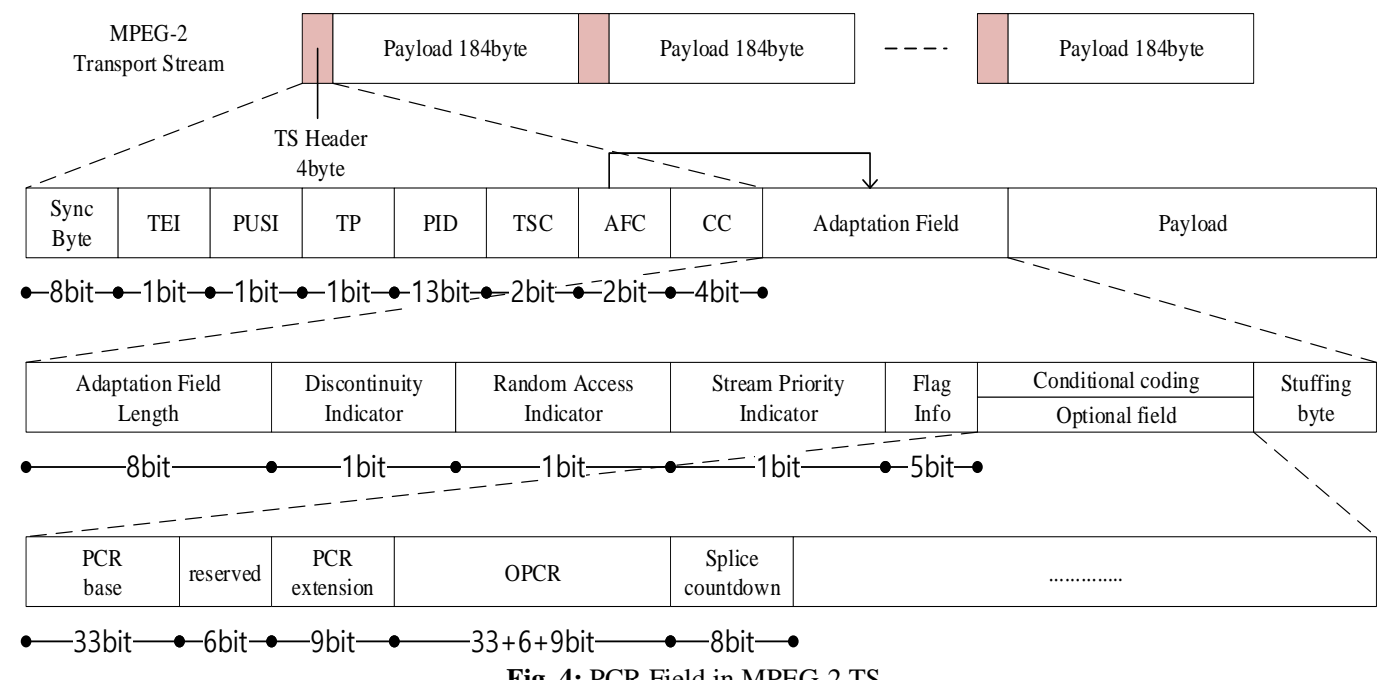

$P C R(i)=P C R_{\text {base }}(i) \times 300+P C R_{\text {ext }}(i)$

$\mathrm{PCR}_{\text {base }}(\mathrm{i})=(($ system_clock_frequency $\times \mathrm{t}(\mathrm{i})) \div 300) \% 2^{33}$

$\mathrm{PCR}_{\text {ext }}(\mathrm{i})=(($ system_clock_frequency $\times \mathrm{t}(\mathrm{i})) \div 1) \% 300$

\subsection{Proposed work}

The proposed method receives the digital stream from the RF tuner or ASI input, analyzes the program information through TS analysis, and finds the PCR value from the MPEG-2 TS. Then, it synchronizes to the MPEG-2 TS PCR location of the input stream between main and spare inputs through the PCR value found. If an error occurs in the main input, spare input would be operated. Otherwise, the main input would be executed.

The PCR synchronization method calculates the PCR value of input 1 and input 2 for each packet using equations 4 and 5 . Then, it stores packets by the PCR difference between input 1 and input 2 and moves to the same PCR packet position at switching time. The following is the PCR synchronization method proposed in this paper.

$P C R_{\text {packet }}=\left(\left(P C R_{\text {Last }}-P C R_{\text {previous }}\right) \div 300\right) \div$

PacketInterval

$P C R_{\text {packet }}(i)=P C R_{\text {packet }} \times$ PacketInterval $(i)$ 
Figure 6 shows the case when the PCR values of an input 1 and an input 2 are exactly the same.

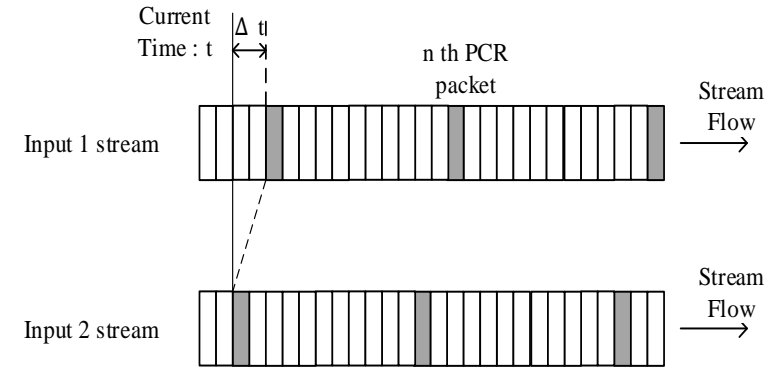

Fig. 5:.Synchronous PCR Method: Packet Flow from Input 1 and 2.

As shown in Figure5, TS packets corresponding to $\Delta \mathrm{t}$ are stored in the buffer when the two input streams are the same. If an input 1 switches to an input 2, the output of a buffered packet is found by input2. Then, there would be no packet loss or PCR delay.

If the PCR values of an input 1 and an input 2 are not the same, the difference between the two input PCR values are calculated and packets are buffered to the PCR difference value. In this case, the input is synchronized as described above. Since the accurate packet synchronization is not possible, packet loss and PCR accuracy error are expected to occur when two inputs are switched. Also, it is limited to double the size of 312 packets due to the limitation of system buffering capacity.

\section{Results and discussion}

The system configuration for the experiment is shown in Figure6. The input 1 stream has lost packet according to the reception environment, and the input 2 stream has used the original stream that received the normal stream. Also, the starting point between the two streams is different and buffered at both inputs 1 and 2 on a packetby-packet basis. The experimental setup is shown in Figure 6 and Figure 7 [7], [8].

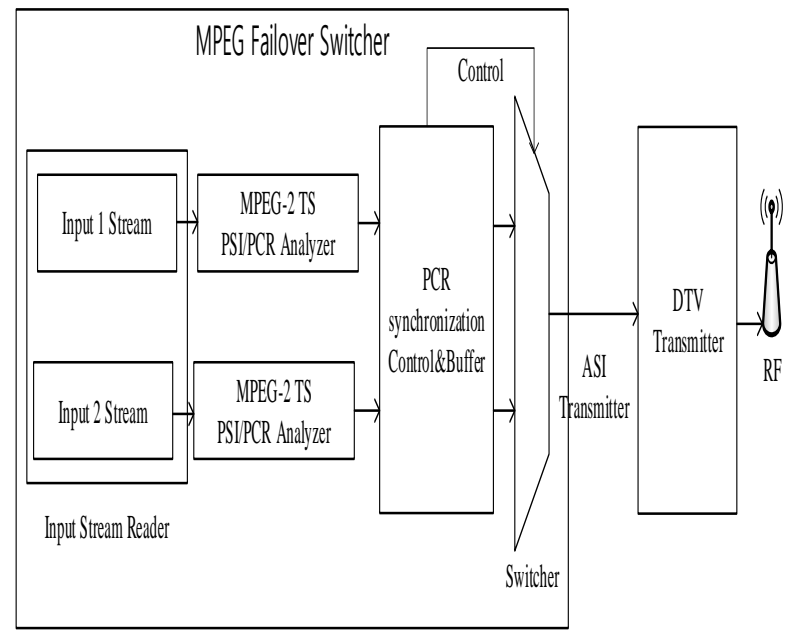

Fig. 6: System Configuration for Experiments

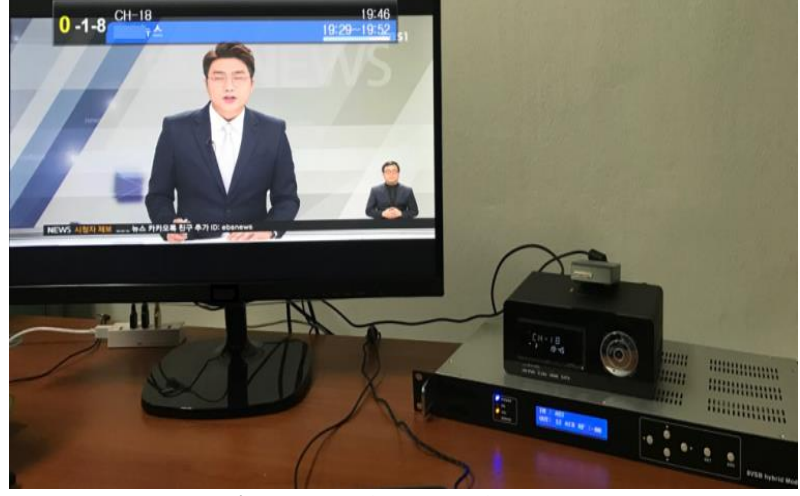

Fig. 7: Experiment Environment.

When the PCR asynchronizes to the input stream due to the lack of performance by PCR monitoring and buffering between input 0 and input 1 , the order of PCR and packet are overlapped or lost. The experimental results are shown in Figure 8.

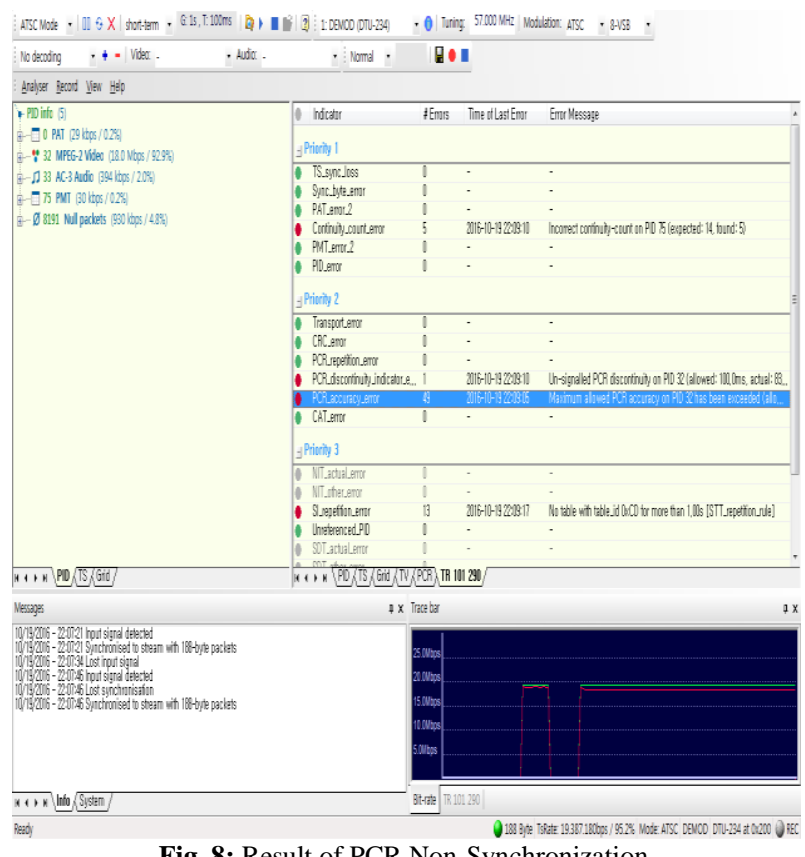

Fig. 8:.Result of PCR Non-Synchronization.

TR101 290 monitoring results in continuity count error, PCR discontinuity, and PCR accuracy error occurred. Moreover, the error rate of TS packet continuity count was high. In this case, video or audio packets are lost [9-12].

For the PCR input synchronization method, processing can be performed without packet loss, because the packet is buffered by its difference between input 1 and input 2. The experimental results are shown in Figure 9. 


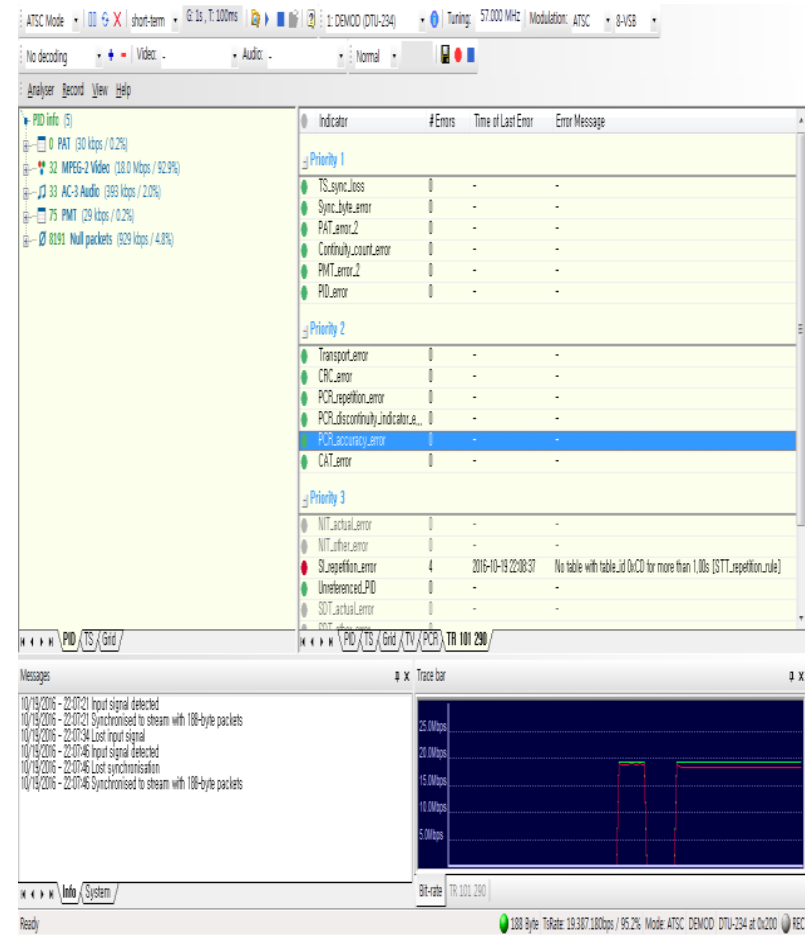

Fig. 9: Result of PCR Synchronization.

\section{Conclusion}

With this proposed method, all inputs can be accurately transferred, although the PCR values of the two inputs are exactly same. If the correct failover switching of the TS packets occurs, it is not possible to know whether failover switching has occurred at the receiver from the broadcast or not. On the other hand, when the PCR value inputs were slightly different, failover switching is performed at a relatively accurate point. In this case, the number of error types and error packets such as continuity count error, PCR discontinuity, PCR accuracy error, and PAT PMT error are decreased by tens of packets. As such, the reduction of error types and packet errors do not require the receiver to initialize the MPEG-2 TS decoder, which can reduce the frequency of video corruption, audio mute, or tick noise.

\section{Acknowledgment}

This work was supported by the Technology Innovation Program (10052464, Development of Multi-Dimensional Visualization Technology for IoT Large Data Interpretation (2nd stage, 1st year)) funded By the Ministry of Trade, Industry \&Energy (MOTIE, Korea).

\section{References}

[1] Yoowon K., Geun-Sik J., "8-VSB Remodulator for Retransmitting the Terrestrial Digital Broadcasting", Journal of Korea Multimedia Society, vol. 13, no. 10, pp.1525-1533, 2010.

[2] https://thorbroadcast.com/product/1-1000mhz-rf-redundancyswitch.html, Rf-redundancy-switch

[3] http://www.etsi.org/deliver/etsi tr/101200 101299/ 101290/01.02.01_60/tr_101290v010201p.pdf, ETSI, Digital Video Broadcasting, Measurement guidelines for DVB systems, ETSI TR $101290 \mathrm{~V} 1.2 .1,2001-05$.

[4] https://www.atsc.org/wp-content/uploads/2015/03/Program-System-Information-Protocol-for-Terrestrial-Broadcast-and-Cable.pdf, ATSC digital television standard, ATSC Standard A/65C, Program \& System Information protocol, 2013

[5] https://www.atsc.org/recommended-practice/a54a-guide-to-the-useof-the-atsc-digital-television-standard-with-corrigendum-no-1/, ATSC digital television standard, Guide to the Use of the ATSC Digital Television Standard, 2003
[6] https://www.iso.org/standard/67331.html, ISO/IEC, Generic Coding of Moving Pictures and Associated Audio Information: Systems, ISO/IEC 13818-1:2013.

[7] https://www.atsc.org/standard/a53-atsc-digital-television-standard/, ATSC, digital television standard; A/53: ATSC Digital Television Standard, Parts 1-6, 2011.

[8] Prendergast D., Caron B., \& Wu Y.,"The implementation of a return channel for ATSC-DTV", IEEE transactions on broadcasting,vol. 53 , no. 2, pp. 521-529, 2007.

[9] Mitra S., Ranjitha M. S., Ravi V., "Video headend video quality monitoring solution", Indian Journal of Science and Technology, vol 8 , no. 19, 2015.

[10] Buitrago M. E., Vargas R. C. G., "Analysis of processing in digital terrestrial television with statistical multiplexing for standards DVBT'. In: Image, Signal Processing, and Artificial Vision (STSIVA), 2013 XVIII Symposium of. IEEE, pp 1-5, 2013.

[11] https://www.dektec.com/products/USB/DTU-234/downloads/DTU234\%20Leaflet.pdf, DekTec, DTU-234 8-VSB / QAM-B RF Probe for USB-2 - DekTec, 2008.

[12] https://www.dektec.com/products/applications/ StreamXpert/downloads/StreamXpert\%202.0\%20and\%20StreamXpert\%20Lite.pdf, DekTec, StreamXpert 2.0 and StreamXpert Lite - DekTec, 2015. 